\title{
CLIP Antenna for Wireless Bluetooth Applications
}

\author{
Hala Elsadek \\ Microstrip Department, \\ Electronics Research Institute, \\ Eltahrir St., Dokki, Giza, Egypt, 12622, \\ Fax: + 2023368584 ,
}

\begin{abstract}
In this paper, a novel design for coplanar waveguide antenna is developed which, consists of two U-shaped slots. The antenna is named Clip. The antenna is designed at central frequency of $2.4 \mathrm{GHZ}$ with input impedance of $50 \Omega$. The antenna dimensions represent $72 \%$ size reduction than conventional microstrip rectangular patch antenna. The measured antenna bandwidth

is about $11 \%$ while its gain is about $17 \mathrm{~dB}$ which; are fairly acceptable in all wireless communication systems. The antenna configuration has bi-directional radiation pattern, while Unidirectional radiation pattern is achieved by using $\lambda_{0} / 4$ reflector with metal plate.

A $2 \times 2$ multi-element sub array is implemented to widen the applications area. The mutual coupling between adjacent elements is low. For more reduction in the mutual coupling orthogonal plane coupling between adjacent elements is introduced. So, the mutual coupling level is reduced to less than $-23 \mathrm{~dB}$ in all coupling planes. Fabrication is done for clip antenna element and array cases. Experimental measurements show very good performance that is highly agreed with simulation results.
\end{abstract}

Keywords: Clip (들essed slot Integrated Coplanar waveguide) antenna, CPW (Coplanar

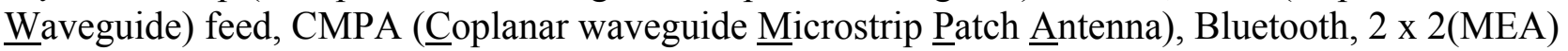

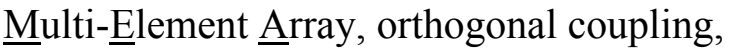

\section{Introduction}

Coplanar waveguide (CPW) fed antennas have been studied widely in recent years especially at microwave frequencies [1]. CPW fed antennas have relatively broader bandwidth than conventional fed microstrip antennas. Coplanar waveguide also has many useful design characteristics such as low radiation loss (especially from feed point), less dispersion, planar configuration (single layer) and amenability for integration with microwave active devices [2]. The CPW fed antenna can be connected to the CPW outputs of semiconductor device circuits. These types of antennas can be used for RF applications because of its easy mounting and integration with other microwave and circuit components. This significantly simplifies the antenna design with components as in most wireless communication systems applications. Lately a coplanar waveguide microstrip patch antennas-have been proposed $[3,4]$. The geometry of a coplanar patch antenna is similar to a loop slot antenna, however the lengths and the behavior of the antenna is similar to the patch antenna rather than loop antenna [5].

CMPA introduces lower losses at millimeter wave frequencies, lower mutual coupling between elements (assuming no unwanted modes exist), close proximity for ground plane for active circuits and lower realestate requirements for antenna elements since the area of the CMPA on the antenna face is about one quarter of similar microstrip antenna element. The resonance of a CMPA antenna occurs when the length of the element perimeter equals one wavelength. However, the resonance 
of the microstrip antenna occurs when the length of the antenna perimeter equals about two wavelengths. Studying microstrip antennas has been the subject of numerous research papers but only few papers deal with the CPW antennas [6]. There are many techniques to improve the characteristics of microstrip antenna. The most common techniques include either stacked multilayer configuration or parasitically coupled single layer configuration. The first technique improves the bandwidth on the cost of increasing complexity and losses due to surface waves. However, the second one opposes the size reduction criteria and makes distortions in the radiation pattern.

Planar antennas fed with coplanar waveguides consisting mostly of slots on a single substrate or slot coupled patches. A new CPW antenna configuration with $\mathrm{CPW}$ feed line called clip is investigated here. The antenna is named clip due to its shape similarity with famous stationery used paper's clips. Clip antenna is a compressed integrated microstrip slot antenna fed by finite width coplanar waveguide to form a semi-rectangular coplanar microstrip patch antenna (CMPA). The antenna is considered as a rectangular microstrip patch antenna with two inter-coupled U-shaped slots on its surface. The entire design is uni-planar with uni-directional radiation pattern. The antenna is designed to work at 2.4 GHZ. Full wave theoretical simulation is done using Ansoft HFSS software Package.

The main disadvantage of the CMPA antennas is the unwanted propagating mode that can be supported between the two ground planes as that shown in figure 1(a). This unwanted mode could introduce spurious or unwanted radiation. Also, the $\mathrm{CPW}$ fed may excite some surface currents flowing across the transverse direction and radiate some unwanted crosspolarized components. Also in array case, this could increase the mutual coupling level between elements. The power propagating away in this mode could reduce the antenna efficiency [6]. The change we suggested here by using two coupled slots, increases the degrees of freedom in the design, hence reduces the effect of this unwanted mode as will be seen from the results. The radiation pattern of the antenna shows very good polarization isolation ratio and also good front to back ratio.
To widen the area of wireless communication applications and to ensure that the unwanted mode has negligible effect on the mutual coupling level, a $2 \times 2$ sub-array is designed and fabricated. Mutual coupling is measured at H, E and Diagonal coupling planes. Orthogonal feed is used to have perpendicular polarization directions for the adjacent elements. This reduces further the levels of mutual coupling to satisfy the uncoupled diversity reception criteria.

The antenna element and the $2 \times 2$ sub array are fabricated using thin film photolithographic technique. The scattering parameters and input impedances are measured using 8719ES vector network analyzer. Very good agreement between the measured and simulated results is achieved in all cases.

\section{Geometry and design procedure}

A clip antenna consists basically of a CPW fed rectangular that consists of two Ushaped slots interfered with each other. This geometry is surrounded by closely spaced ground plane as shown in figure 1 , which represents the 3D shape as well as the photo of the antenna.

The CPW feed and the U-shaped slots are located on the same surface of a conductor backed microwave substrate. Energy from the smaller U-shaped slot is coupled to the larger Ushaped slot. Controlling the amount of this coupling is done by changing the arms' lengths of the two slots or the inter-distance between them (d). By this method, one can both control the antenna matching and at the same time avoid the unwanted propagating modes. We find that the best matching occurs when the two slots are of same gap width $(\mathrm{g})$ and $\mathrm{g}=\mathrm{d}$ at the same time. The characteristics impedance of the CPW feed line is $50 \Omega$ in order to match the measuring system.

The antenna length $\mathrm{L}$ is $30 \mathrm{~mm}(0.24 \lambda \mathrm{o})$ and width $\mathrm{W}$ is $16 \mathrm{~mm}(0.128 \lambda \mathrm{o})$. The gap width of the U-slots $\mathrm{g}$ is $3 \mathrm{~mm}(0.024 \lambda \mathrm{o})$. The antenna resonates at $2.4 \mathrm{GHz}$. The antenna size is reduced by $72 \%$ as compared to conventional patch antenna of size $\lambda / 2 \times \lambda / 2$ [7]. It is worth here to mention that a wider antenna bandwidth can be achieved if we make the two arms of the U-slots with unequal widths hence; in this case 
two staggered modes are excited creating a wide bandwidth structure. Also a more compact antenna size can be introduced by applying fractal concept on the radiation side of the Uslots [8]. The author has started the research in this subject [9].

As shown in figure 1, a quarter wavelength reflector is added for uni-directional radiation pattern. The thickness of this reflector is determined from the following equation:

$$
h_{1} / \sqrt{\varepsilon_{r 1}}+h_{21} / \sqrt{\varepsilon_{r 2}}+\ldots \ldots . .+h_{n} / \sqrt{\varepsilon_{r n}}=\lambda_{0} / 4
$$

The designed clip antenna is implemented in a $2 \times 2$ sub array as shown in figure 2(a). Multi element array MEA is desired since in a Raleigh fading environment, the reliability of wireless communication can be improved and the capacity can be increased by using MEA [10]. The array inter-element distance-edge to edge- is $0.16 \lambda_{\mathrm{o}} \mathrm{x} 0.07 \lambda_{\mathrm{o}}$ in $\mathrm{x}$ and $\mathrm{y}$ directions, respectively. The coplanar waveguide feed reduces the radiation loss due to the coplanar waveguide feed line structure. Mutual coupling study shows that using coplanar patch antenna reducing the mutual coupling than similar microstrip array case with same conditions. This could be attributed due to the slot structure. The mutual coupling between any two linear polarized antennas oriented in parallel polarization [E and $\mathrm{H}$ plane] is reduced by using low dielectric material as duriod $\varepsilon_{\mathrm{r}}=2.2$ which is used here [10]. However coplanar patch antenna have noticeable reduced mutual coupling than normal patch antenna. The reduction is by about $3-6 \mathrm{~dB}$ at same inter-element distance as shown in table 1 . The inter-element spacing is selected to have a compact array size. The mutual coupling is further reduced at same interelement spacing when adjacent elements are oriented in orthogonal polarizations of O-plane as shown in figure 2(b). The mutual coupling level is reduced in this case by further $10-15 \mathrm{~dB}$ [10]. Our clip antenna array satisfies the diversity reception criteria that the mutual coupling in all cases is $\leq-15 \mathrm{~dB}$ [11].

The antenna element and array are simulated and fabricated and the S- parameters and radiation patterns are measured as in the following section.

\section{Simulation and experimental}

\section{results}

The single Clip antenna element and the 2 x 2 sub-array are simulated using HFSS commercial software package based on finite element method. A low dielectric constant material of duriod with $\varepsilon_{\mathrm{r}}=2.2$ is used in this work. The substrate height is $1.5 \mathrm{~mm}$. Background conductor is used to obtain unidirectional radiation pattern. The CPW feed line is designed to be of $50 \Omega$ in order to match the measurement system. Dimensions of the CPW are calculated by using closed-form formulas given in [12] and confirmed by the simulation. The resonant frequency is $2.4 \mathrm{GHZ}$ with bandwidth of $4.5 \%$ at $-10 \mathrm{~dB}$ return loss, which is wider than microstrip rectangular patch antenna. The antenna gain is $17.4 \mathrm{~dB}$. The simulated radiation pattern of the antenna in both parallel E-plane $(\mathrm{phi}=\mathrm{o})$ and perpendicular $\mathrm{H}$ plane $(\mathrm{phi}=90)$ are illustrated in figure $3(\mathrm{a})$ and (b), respectively.

The antenna is fabricated using wet thin film photolithographic technique. Reflection coefficient $S_{11}$ is measured using 8719 ES vector network analyzer. Figure 4 shows the simulation and measured reflection coefficient of the clip single element antenna. The experimental bandwidth is more than $10 \%$. This could be attributed due to the connection of the feed port as that illustrated in antenna photo of figure 1. The parallel component (coplanar) radiation and the perpendicular component (cross-polar) radiation are measured in both E-and H-planes, respectively. Figure 5 shows the experimental radiation pattern with quarter wavelength reflector for uni-directional radiation. Good agreement is found between the measured and simulated results.

As discussed before in section 2, the main disadvantage of CPW structures is the unwanted propagation mode that can propagate through the edge of the ground plane and generate spurious or unwanted radiation, which degrades the radiation pattern. From figures 3 and 5 , it is clear that the radiation pattern is approximately omni directional with good front-to-back ratio. The cross polarization level is less than $-25 \mathrm{~dB}$. This result verifies that the clip antenna design has no evidence of these unwanted propagation modes. 
The $2 \times 2$ clip sub-array is simulated in parallel H-plane arrangement and in orthogonal plane arrangement. The array is then fabricated on same duriod dielectric material with same inter-element distance together with a $2 \times 2$ rectangular microstrip array with same material and design conditions. The microstrip antenna elements are fed using microstrip lines. Table 1 illustrates the experimental comparison between the mutual coupling scattering parameters $S_{i j}$ for parallel plane arrangements.

Table1: Comparison between the measured values of mutual coupling parameters in parallel plane arrangements for a $2 \times 2$ array of Clip antenna and $2 \times 2$ array of rectangular microstrip antenna. The measurements are at resonance frequency $2.4 \mathrm{GHz}$.

\begin{tabular}{|l|l|l|}
\hline$S_{i j}$ in dB & $\begin{array}{l}\text { Microstrip } \\
\text { rectangular } \\
\text { patch antenna }\end{array}$ & Clip antenna \\
\hline $\begin{array}{l}\text { E-plane } \\
\text { coupling }\end{array}$ & -18 & -23.6 \\
\hline $\begin{array}{l}\text { H-plane } \\
\text { coupling }\end{array}$ & -33.2 & -39.2 \\
\hline $\begin{array}{l}\text { D-plane } \\
\text { coupling }\end{array}$ & -41 & -44 \\
\hline
\end{tabular}

From the table, it is clear that the clip antenna configuration reduces the mutual coupling level than the conventional microstrip antenna in all coupling plane directions.

Further mutual coupling reduction is done by re-arranging the elements to be in orthogonal directions as that shown in figure 2(b). The interelement distances are kept the same. The mutual coupling is reduced by about $8 \mathrm{~dB}$ in average. This is reasonable as in O-plane case if one in the two adjacent ports receives (transmits) horizontal linear polarization; the other one receives (transmits) vertical linear polarization [9]. Again we didn't notice any effect of the unwanted propagating mode on the mutual coupling level. From the above discussion, it is clear that the clip antenna array has the advantage of compact size since the interelement distance can be reduced than that in a similar case of conventional microstrip antenna without affecting the mutual coupling level.

\section{4- Conclusion}

We have introduced a new co-planar microstrip antenna configuration with CPW line feed The new design has more degrees of freedom to get more optimum design. The design parameters are not only the patch dimensions but also the slots. The CPW line feed reduces the feed losses to conductor loss only. This feeding type enables the antenna connection to microwave circuits easily for wireless communication applications. The antenna characteristics show the similarity between clip antenna and microstrip rectangular patch antenna However, clip antenna offers several advantages over conventional microstrip antenna as the planar configuration, lower losses, and lower space requirement since the antenna area is about one quarter of the microstrip antenna area. The mutual coupling study in the $2 \times 2$ array illustrates the reduced coupling levels comparing to the microstrip antenna case. The orthogonal plane coupling makes further reduction to the coupling level. This demonstrates that clip antenna array with O-plane arrangement is a successful candidate for a compact size array with diversity reception applications in wireless communication systems. Experimental measurements show very good agreement with simulation results in both single element and array cases.

\section{References}

[1]K.F. Tong, K.Li, T. Matsui and M. Izatsu, "Wideband coplanar waveguide fed coplanar patch antenna", IEEE MTT-S International Symposium Digest, 2001, pp: 406:409.

[2] Guiping Zheng, Atef. Z. Elsherbeni and Charles E. Smith, "A coplanar waveguide Bow-Tie aperture antenna", IEEE AP-S International Symposium Digest, San Antonio, Texas, June 2002.

[3] K.Li,H. Cheng, T. Matsui and M. Izatsu,"Coplanar patch antenna", IEEE MTT-S International Symposium Digest, 2001, pp:402:405. [4] http://www.nasatech.com/Briefs/May99/LEw16666 html, NASA technical report on "Finite width coplanar patch antenna", May 99.

[5]Rainee N.Simons, Richard Q.Lee and Kurt A. Shalkhuser, "Finite width coplanar waveguide patch antenna with vertical feed through interconnect", IEEE 
AP-S International Symposium Digest, 1996, pp: 1338:1341.

[6] John Litva, Chen Wu, Zheqiang Bi and Kell We, "Some considerations for coplanar waveguide antennas", IEEE AP-S International Symposium Digest, 1992, pp: 491-494.

[7] K. Li, C. H. Cheng, T. Matsui and M. Izytsu, "Coplanar patch antennas: Principle, simulation and experiment", IEEE AP-S International Symposium Digest, Boston, Massachusetts, June 2001.

[8] Hala Elsadek, Dalia Nashaat and Hani Ghali, "Multiband miniaturized PIFA for compact wireless communication Applications", Microwave and optical technology letters, submitted.

[9] Dalia M. Nashaat, Hala A. Elsadek, Member IEEE and Hani Ghali, "Single feed compact quad band PIFA for wireless communication applications", IEEE Transactions on Antenna and Propagation, under press.

[10] R.R. Ramirez and Franco De-Flaviis, "A mutual coupling study of linear and circular polarized microstrip antennas for diversity wireless systems", IEEE Transactions on Antennas and Propagation, vol.51, No. 2, Feb. 2003, pp:238-249.

[11] Koichi Tsunekawa and Kenichi Kagoshima, "Analysis of correlation coefficient of built-in diversity antennas for portable telephone", Proceedings of IEEE Antenna and Propagation, 1990, pp: 543:546.

[12] K.C. Gupta, R. Grag, I. Bahl and P. Bhatia, "Microstriup lines and slotlines", Second edition, Artech house, Norwood, MA, 1996.

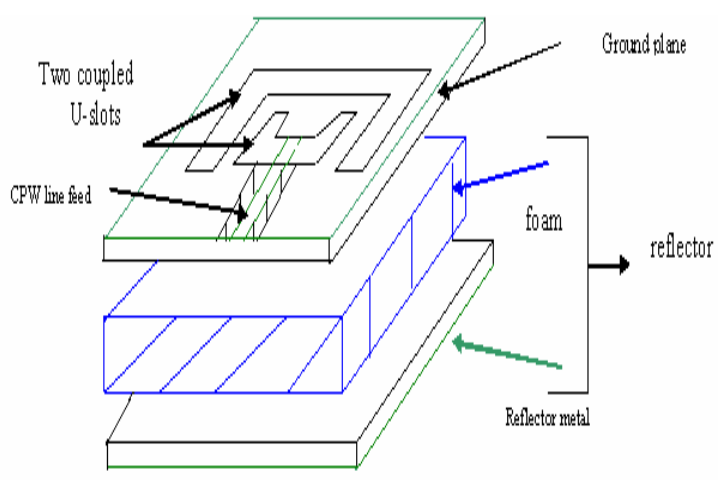

(a)

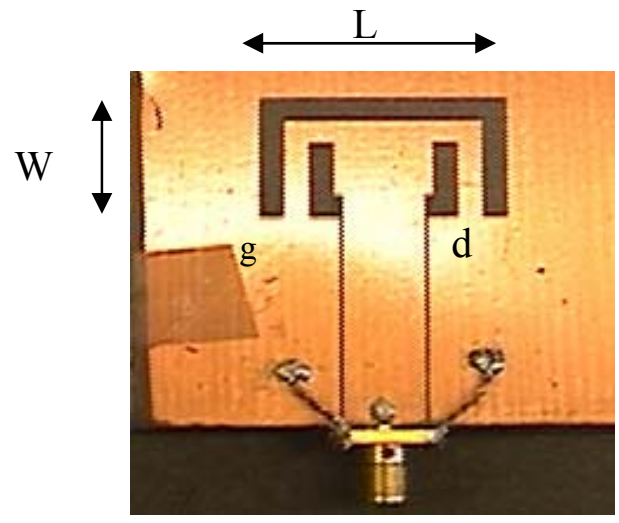

(b)

Fig.1 (a) 3D configuration of clip antenna and (b) photo of fabricated antenna

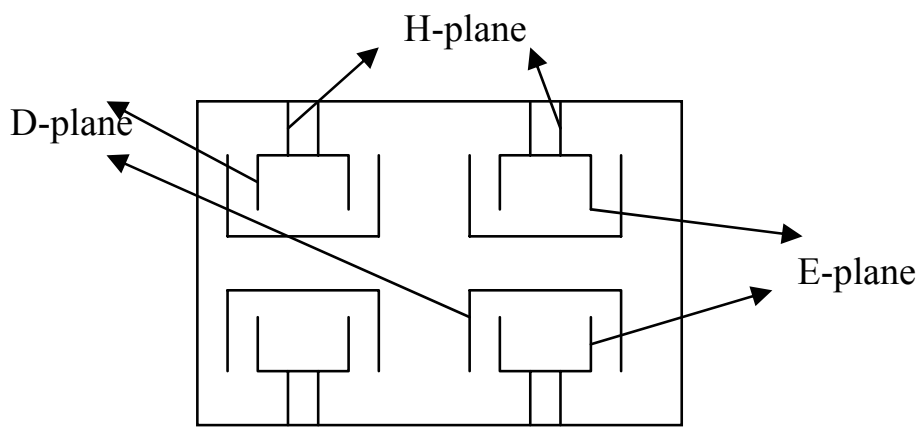

(a) Parallel plane



(b)Orthogonal plane Arrangement

Fig. 2: 2x2 sub array of clip antenna (a) parallel plane arrangement and (b) orthogonal plane arrangement. 

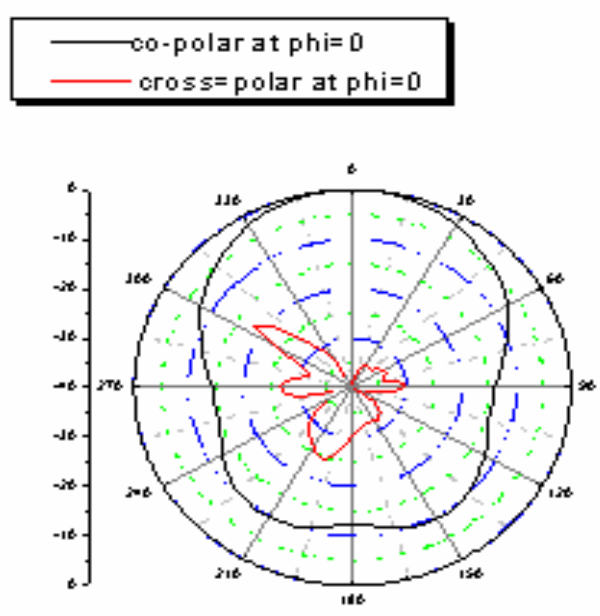

(a)



(b)

Fig.3: Simulated radiation pattern of single clip antenna element (a) parallel plane at phi $=0^{\circ}$ and (b) perpendicular plane at $\mathrm{phi}=90^{\circ}$

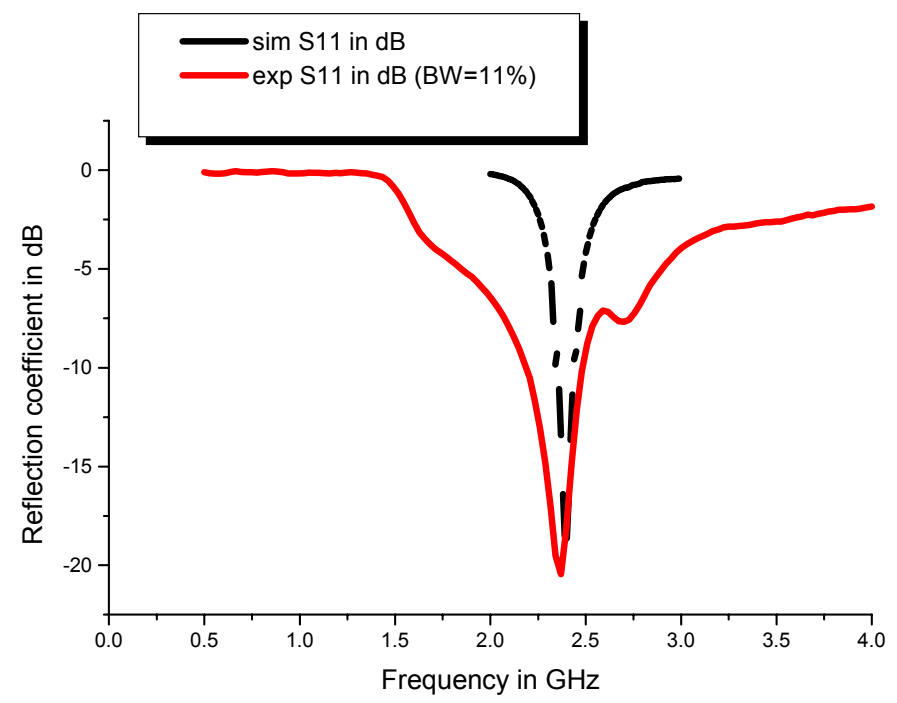

Fig.4: Comparison between the simulated and experimental reflection coefficient of single element clip antenna.


Fig. 5: Experimental radiation pattern of single clip antenna element using quarter wavelength reflector for uni-directional radiation (a) parallel plane at $\mathrm{phi}=0^{\circ}$ and (b) perpendicular plane at $\mathrm{phi}=90^{\circ}$. 